\title{
Seroprevalence of Hepatitis E Virus in Autoimmune Hepatitis Patients in the Netherlands
}

\author{
Nicole M.F. van Gerven', Annemiek A. van der Eijk², Suzan D. Pas², Hans L. Zaaijer ${ }^{3}$, Ynto S. de Boer ${ }^{1}$, Birgit I. Witte ${ }^{4}$, \\ Carin M.J. van Nieuwkerk ${ }^{1}$, Chris J.J. Mulder ${ }^{1}$, Gerd Bouma ${ }^{1}$, Robert A. de Man ${ }^{5}$
}

On behalf of the Dutch Autoimmune Hepatitis Study Group ${ }^{\star}$

\author{
1) Department of \\ Gastroenterology and \\ Hepatology, VU University \\ Medical Centre, Amsterdam \\ 2) Department of Virology, \\ Erasmus Medical Centre, \\ Rotterdam \\ 3) Department of Blood- \\ borne infections, Sanquin \\ Blood Supply Foundation, \\ Amsterdam \\ 4) Department of \\ Epidemiology and \\ Biostatistics, VU University \\ Medical Centre, \\ Amsterdam \\ 5) Department of \\ Gastroenterology and \\ Hepatology, Erasmus Medical \\ Centre, Rotterdam \\ The Netherlands
}

\begin{abstract}
Background \& Aims: In recent years chronic courses of hepatitis E virus (HEV) infection have been described in immunosuppressed individuals. This may implicate a potential role for HEV in the development of autoimmune diseases, including autoimmune hepatitis (AIH). Here we investigated the prevalence of HEVantibodies in $\mathrm{AIH}$ patients in an endemic Central European country.

Methods: HEV-specific immunoglobulin G (IgG) and HEV RNA were determined in 354 and 377 AIH patients, respectively. Clinical characteristics and disease outcome parameters were retrospectively collected. Results: No HEV viraemic patients were identified in this cohort. A total of $106 \mathrm{AIH}$ patients (29.9\%) tested positive for anti-HEV IgG, and this figure was slightly higher compared to the prevalence in a reference cohort including 5,329 healthy Dutch blood donors (26.7\%; P>0.05).

Conclusion: This is the largest study on the association between HEV infection and AIH. Apparently silent $\mathrm{HEV}$ infection is present in a significant proportion of AIH patients, yet appears not to have significant clinical repercussions in this immune compromised group of patients. Nevertheless, since acute hepatitis E may present with histological and biochemical features of AIH, the possibility of a (concomitant) HEV infection should be considered in this category of patients.
\end{abstract}

Key words: hepatitis E virus infection - autoimmune hepatitis - anti-HEV IgG - prevalence.

Abbreviations: AIH: autoimmune hepatitis; ALT: alanine aminotransferase; SMA: anti smooth muscle antibodies; ANA: antinuclear antibodies; PBC: primary biliary cirrhosis; AMA: anti mitochondrial antibodies; PSC: primary sclerosing cholangitis.

\section{INTRODUCTION}

Autoimmune hepatitis (AIH) is an uncommon chronic inflammatory disease in which loss of immunotolerance against hepatic tissue is presumed. It is characterized by a female predominance, histological features of periportal hepatitis in the absence of viral markers, hypergammaglobulinemia and the presence of serum autoantibodies [1].

Diagnostic scoring systems have been developed that support the diagnosis in the majority of patients [2]. A simplified scoring system with four variables was recently developed that enhances applicability in daily practice. In this scoring system points were subtracted if viral hepatitis was present [3]. Genetic background, hormones and environmental agents are persistently listed as contributing factors to AIH development. Exogenous factors, such as viruses and drugs, have been proposed as triggers for AIH. There have been occasional cases presenting shortly after documented infection with hepatitis A virus [4, 5], cytomegalovirus [6], Epstein Barr virus (EBV) [7] and hepatitis E virus (HEV) $[8,9]$. This supports the evidence of the initiating role of hepatitis viruses in the development of AIH and other autoimmune diseases [10].

Hepatitis E virus infection is an important cause of acute clinical hepatitis in adults throughout Asia, the Middle East and Africa. Recent data suggest that HEV could be more widespread than previously thought and that the disease can also be found in more developed countries. The course of HEV infection can vary substantially between different individuals. Although most infections follow a clinically silent asymptomatic course, other patients may develop severe hepatitis that can progress to fulminant hepatic failure 
[11]. In recent years, a chronic course of HEV infection has been described in immunosuppressed individuals including AIH patients who are taking immunosuppressive drugs [12]. Studies on the prevalence of HEV in AIH patients are scarce and performed in small sample sets. A recent study by Pischke et al. involving $208 \mathrm{AIH}$ patients found that $\mathrm{AIH}$ patients are more likely to test anti-HEV positive and it is recommended to rule out $\mathrm{HEV}$ infections, especially in $\mathrm{AIH}$ patients not responding to immunosuppressive treatment [13]. More clinical observations are required to reveal the possible link between HEV and AIH.

Here we aimed to further investigate the potential association between HEV and AIH by assessing the prevalence of HEV RNA and HEV antibodies in a large cohort of Dutch AIH patients and controls.

\section{PATIENTS AND METHODS}

\section{Patient population}

The AIH patients were identified by the Dutch AIH Study Group consortium (http://www.autoimmuunhepatitis.nl) involving the gastroenterology and hepatology departments from 6 academic and 19 general hospitals in the Netherlands and has been described in details elsewhere [14]. AIH patients were identified by their treating physicians and by searching the database for international classification of diseases (ICD) codes. The search was performed in local diagnostic registers in the departments of gastroenterology and hepatology as well as internal medicine. In all patients clinical and biochemical parameters were assessed to exclude other aetiologies such as alcohol, drugs and metabolic disorders. Viral hepatitis (B and C) was excluded by serological testing. If performed, liver biopsy was used to establish diagnosis and the presence of fibrosis and cirrhosis. We recorded manifestations of overlap syndromes with primary biliary cirrhosis (PBC) and primary sclerosing cholangitis (PSC) in the presence of AIH if available. For $\mathrm{PBC}$, these criteria consisted of anti-mitochondrial antibody (AMA) titres higher than 1:80 and typical histological findings, whereas manifestations of PSC were recorded in cases of typical histological and radiological findings. Diagnostic scores were determined according to the revised original International Autoimmune Hepatitis Group (IAIHG) criteria [2]. Available data on induction and maintenance therapy, as initiated and recorded by the treating physician, were retrospectively collected from the patient hospital records. Similarly, both clinical response to induction therapy and the occurrence of a relapse after treatment withdrawal were scored as assessed by the treating physician. In the 6 academic centres when a liver transplantation had been performed and when a patient developed a hepatocellular carcinoma (HCC), it was documented. Prior to the start of the study, institutional review board approval to carry out the study was obtained in all participating centres.

\section{Control population}

Findings were compared with a control group of healthy blood donors from a recent study on the prevalence of HEV in the Netherlands $(n=5,329)$ [15].

\section{HEV RNA detection}

From September 2010 until January 2011 serum samples were obtained from each subject. The samples were aliquoted and stored frozen at $-80^{\circ} \mathrm{C}$. HEV RNA was tested by an internally controlled quantitative real-time Polymerase Chain Reaction (RT-PCR,) as described elsewhere [16]. In one serum sample the volume was not sufficient to test for HEV RNA. The RT-PCR had a lower limit of detection (LOD, 95\% cut-off) of $143 \mathrm{IU} / \mathrm{ml}$ HEV RNA, as determined by the first World Health Organization (WHO) standard for HEV RNA nucleic acid testing-based assays (6329/10, Paul Ehrlich Institute, Germany) [17]. Due to the limited sample volume, samples were diluted $10 \mathrm{x}$ prior to testing, thereby increasing the LOD to $1430 \mathrm{IU} / \mathrm{ml}$.

\section{HEV-specific antibody detection}

In addition, the samples were collected to determine HEV IgG seroprevalence with subsequent testing for HEV-specific immunoglobulin $\mathrm{G}$ ( $\mathrm{IgG}$ ) in serum or EDTA plasma samples. In 23 serum samples the volume was not sufficient to test anti-HEV IgG. A commercially available enzyme-linked immunosorbent assay (ELISA; Wantai, Beijing, China) was used according to the manufacturer's instructions.

Patients tested positive (optical density, $O D>1.1$ ), intermediate $(0.9<\mathrm{OD}>1.1)$ or negative $(\mathrm{OD}<0.9)$. In clinical practice when patients test intermediate, a control sample is done after two weeks to ensure positivity. Since no follow up sample was collected in this study, patients that tested intermediate were marked negative.

\section{Statistical analysis}

Summary statistics for categorical variables are expressed as numbers (percentages). Quantitative variables are described as medians with their range if not normally distributed. Statistical analysis was performed using Statistical Package for Social Sciences (SPSS) version 20, IBM, corp, Armonk NY. Depending on the distribution, parametric and nonparametric tests including Mann Witney test were used to test for differences within and between groups. The chi square test was used for the comparison between the results of the recent study and controls (adapted from Slot et al.) [15]. A two sided P-value < 0.05 was considered statistically significant.

\section{RESULTS}

\section{Cohort characteristics}

The AIH cases consisted of 66 (17\%) males and 311 (83\%) females with a mean age at diagnosis of 45 years $(S D \pm 18)$. The median IAIHG diagnostic score was 18 (Interquartile range, IQR: 15-21) (18). Based on the antibody profile, the large majority (95\%) had type $1 \mathrm{AIH}$, whereas a small minority had positive anti-LKM 1 antibodies and was thus classified as type $2 \mathrm{AIH}$. Evidence for a clinical overlap-syndrome with PBC (AIH-PBC) and PSC (AIH-PSC) was found in 23 (7\%) patients and $13(3 \%)$ patients, respectively.

Prevalence of HEV RNA and HEV-specific antibodies

None of the 377 AIH patients tested positive for the presence of HEV RNA. In addition, patients were screened 
for IgG antibodies against HEV. In 23 patients the serum or plasma volume were insufficient for this analysis. Five patients tested intermediate and as mentioned before were marked negative. Screening of $354 \mathrm{AIH}$ patients for the presence of HEV antibodies revealed 106 patients who were positive for anti-HEV IgG, which represents a seroprevalence of $29.9 \%$. This is slightly higher when compared to the prevalence of $26.7 \%$ in the Dutch population [15]; however, this difference was not significant $(\mathrm{P}=0.18)$.

In both the general Dutch population as well as in the AIH patients, the anti-HEV IgG seroprevalence strongly increased with age $(\mathrm{P}=0.01)$. Subanalysis in age-adjusted groups (grouped in 10-year cohorts) did not reveal differences between $\mathrm{AIH}$ patients and the general population (Fig. 1).

In the small group of 18-30 years AIH patients, the seroprevalence of anti-HEV IgG was $21.2 \%$ compared to $12.6 \%$ in the general population (difference not significant $\mathrm{P}=0.12$ ).

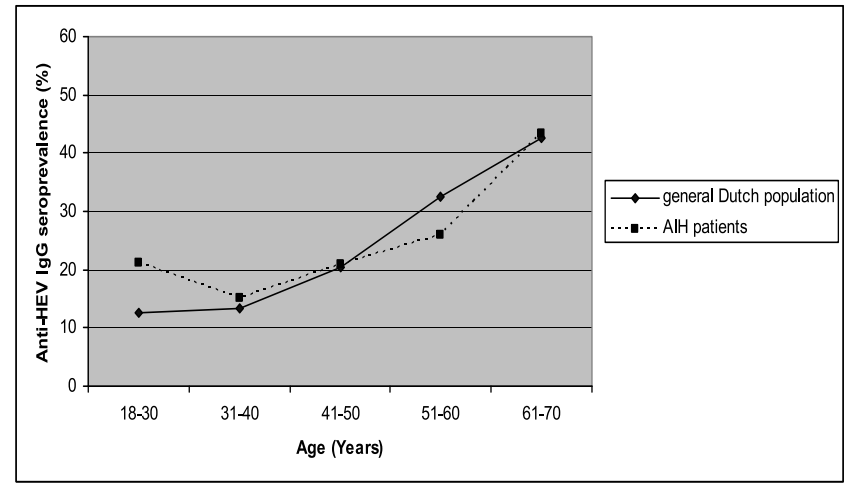

Fig. 1. Anti-HEV IgG seroprevalence in the 10-year age groups of AIH patients and the general population in the Netherlands.

\section{Association of anti-HEV IgG with clinical features}

Anti-HEV IgG positivity was associated with age of onset (median age 55 vs. 44 years) $(\mathrm{P}=0.01)$, but not with gender. Anti-HEV IgG was not associated with a higher IAIHG score at diagnosis. Median alanine aminotransferase (ALT) levels, IgG and alkaline phosphatase (ALP) levels at time of diagnosis were not associated with anti-HEV IgG. Antinuclear antibodies (ANA) and liver kidney microsomal antibodies (LKM-1) positivity was equally distributed among the anti-HEV IgG positive and negative patients. Smooth muscle antibodies (SMA) were associated with anti-HEV IgG $(\mathrm{P}=0.03)$.

We could not identify a significant relationship between anti- HEV IgG and the occurrence of AIH-PSC and AIH-PBC. Anti-HEV IgG were not associated with cirrhosis and fibrosis. Patients with anti-HEV IgG did not receive medication more often and no association between anti-HEV IgG and response to treatment was found.

Evidence of hepatocellular carcinoma (HCC) was observed in one patient who was anti-HEV IgG negative. A liver transplantation had not been performed in any of the patients. All characteristics are summarized in Table I.

\section{DISCUSSION}

In this large multicentre study, we demonstrated that the prevalence of anti-HEV IgG in AIH patients is similar to that in age-matched healthy controls. In addition, we did not identify chronically HEV-infected patients that were erroneously classified as AIH.

Reports on anti-HEV IgG seroprevalence in AIH patients date back to the 1990s $[9,19]$. In 1997, Le Cann et al. studied the sera of $52 \mathrm{AIH}$ patients in the United States; anti-HEV IgG

Table I. Patient characteristics in anti-HEV IgG positive and negative AIH patients at diagnosis

\begin{tabular}{|c|c|c|c|c|}
\hline Features & Anti-HEV IgG negative & Anti-HEV IgG positive & $(\mathrm{N})$ & $\mathrm{P}$ \\
\hline & $\mathrm{N}=248$ & $\mathrm{~N}=106$ & & \\
\hline Gender (female); n (\%) & $208(83 \%)$ & $88(83 \%)$ & 354 & 0.9 \\
\hline Median age; years (IQR ) & $44(26-58)$ & $54(41-63)$ & 354 & 0.01 \\
\hline Median ALT; IU/L (IQR) & $401(179-901)$ & $361(199-900)$ & 354 & 0.3 \\
\hline Median IgG; g/L (IQR) & $22.3(16.2-31.3)$ & $22.5(18.1-29.2)$ & 347 & 0.9 \\
\hline Median ALP; IU/L (IQR) & $135(94-203)$ & $181(121-255)$ & 351 & 0.5 \\
\hline Median IAIHG score (IQR) & $18(15-21)$ & $18(16-21)$ & 339 & 0.6 \\
\hline SMA positive; $\mathrm{n}(\%)$ & $95(41 \%)$ & $54(54 \%)$ & 332 & 0.03 \\
\hline ANA positive; $\mathrm{n}(\%)$ & $139(60 \%)$ & $56(56 \%)$ & 332 & 0.5 \\
\hline Cirrhosis/fibrosis (biopsy); n (\%) & $18 / 105(10 \% / 56 \%)$ & $13 / 51(15 \% / 58 \%)$ & 276 & $0.2 / 0.7$ \\
\hline Concomitant autoimmune disease; $\mathrm{n}(\%)$ & $46(20 \%)$ & $22(22 \%)$ & 331 & 0.9 \\
\hline PBC overlap; n (\%) & $17(7 \%)$ & $7(7 \%)$ & 335 & 0.9 \\
\hline PSC overlap; n (\%) & $8(3 \%)$ & $3(3 \%)$ & 331 & 0.9 \\
\hline Portal hypertension; n (\%) & $5(2 \%)$ & $0(0 \%)$ & 354 & 0.3 \\
\hline HCC; n (\%) & $1(0.4 \%)$ & $0(0 \%)$ & 354 & 1 \\
\hline Liver transplantation and waiting list; $\mathrm{n}(\%)$ & $0(0 \%)$ & $0(0 \%)$ & 354 & 0.08 \\
\hline Mono/combination treatment; $\mathrm{n}(\%)$ & $90 / 100(39 \% / 43 \%)$ & $45 / 40(50 \% / 44 \%)$ & 331 & 0.4 \\
\hline
\end{tabular}

IQR: Interquartile range; ALT: alanine aminotransferase; ALP: Alkaline phosphatase; AIHG: International autoimmune hepatitis group; SMA: smooth muscle antibodies; ANA: antinuclear antibodies; PBC: Primary biliary cirrhosis; PSC: Primary sclerosing cholangitis; HCC: Hepatocellular carcinoma. 
was found in 7 patients (13\%). However, systematic large scale studies on the prevalence of HEV in AIH patients have not been reported so far. In addition, these studies were hampered by the fact that they were executed in single centre or tertiary referral hospitals.

There is no gold standard for HEV antibody testing, which impairs the ability to compare different studies. In a recent study by Pischke et al. involving 208 German AIH patients, the seroprevalence of anti-HEV IgG was higher (7.7\%) than in the healthy controls (2\%) [12]. The MP/Genelabs assay used in this study was also employed in a recent study in 7,072 Dutch samples collected in 2006 and 2007, in which a seroprevalence of only $2.6 \%$ was found [20]. When compared to the Wantai assay used in this study, the MP/Genelabs assay was shown to be considerably less sensitive in PCR-proven HEV cases which explains the considerable higher seroprevalence found in this study $[21,22]$. In the study by Pischke et al., in a subgroup of AIH patients $(n=123)$ anti-HEV was tested using the Wantai assay. When using the Wantai assay 33\% AIH patients tested anti-HEV positive, confirming the Wantai assay to be more sensitive [13]. In Austria, using the same assay a seroprevalence of $22.7 \%$ was found [23]. Other studies have shown that the seroprevalence among the general population varies widely. Thus, while using the same antibody assay (Wantai) as employed in this study, a seroprevalence of $16 \%$ was found in a cohort of healthy donors in the south-west of the United Kingdom $(n=500)$ [24], whereas this percentage was as high as $53 \%$ in south-western France $(n=512)$ [21]. Our findings presented in this study are in line with these earlier observations. In our study $29.9 \%$ AIH patients tested positive for anti-HEV using the Wantai assay. Although not significant, this is slightly higher when compared to the prevalence of $26.7 \%$ in the general Dutch population [15]. Based on these results, an association between $\mathrm{AIH}$ and HEV cannot be confirmed, although due to the slightly higher prevalence in $\mathrm{AIH}$ patients it cannot be completely ruled out.

As mentioned in earlier studies there is a striking age related seroprevalence, while HEV IgG positivity was associated with age of onset (median age 55 vs. 44 years). This may simply reflect a long-standing stable situation, in which people are evenly exposed to HEV in the course of their life.

In the small group of AIH patients aged 18-30 years, the seroprevalence of anti-HEV IgG was $21.2 \%$ compared to $12.6 \%$ in the general population; this difference was not significant (perhaps due to the small size of this subgroup). One possible explanation for the elevated seroprevalence in young $\mathrm{AIH}$ patients is that previous HEV infection in some of these young patients might have triggered the later development of AIH. The current pathogenic concept of $\mathrm{AIH}$ is that a genetically susceptible subject is exposed to an environmental agent, which triggers an autoimmune process. Various viruses have been supposed to be the triggering agent of AIH, but for most of these no compelling evidence has been found [5]. Some studies have suggested that EBV could trigger the process, whereas others have found evidence for suggested other viruses such as initiators of the disease [25].

A similar concept has been proposed for neurologic manifestations such as Guillain Barré syndrome and Neuralgic Amyotrophy in association with HEV infection [26, 27].
While based on the observations in this cross-sectional study, it cannot be ruled out completely that an HEV infection may have triggered immune events leading to the manifestation of AIH in a subset of patients; a role for a chronic persistent infection appears unlikely.

Based on these results we cannot rule out that in younger $\mathrm{AIH}$ patients the HEV seroprevalence is higher compared to that of the general population; other studies are necessary to confirm this.

Acute hepatitis E presents with histological and biochemical features of $\mathrm{AIH}$ and thus acute HEV infection may be misclassified as de novo onset of $\mathrm{AIH}$ and treatment with immunosuppressants in acutely infected patients may accelerate histological progression. Since HEV infection might be life threatening in immunocompromised patients [16] and most AIH patients will be treated with low doses of steroids with or without azathioprine, routine serological testing of $\mathrm{HEV}$ infection should be strongly recommended in the initial work-up of AIH. Similarly, AIH patients with unexplained elevated liver enzymes should also be tested for HEV-RNA.

\section{CONCLUSION}

No AIH patients with active HEV were found in our study and we did not identify chronically HEV-infected AIH patients. In addition, the seroprevalence of anti-HEV IgG, suggesting past exposure to HEV is almost similar in Dutch AIH patients to that in the general Dutch population. Despite the absence of active HEV disease, awareness of the possibility of HEV infection in patients diagnosed with AIH should be kept in mind.

\section{Conflicts of interest: none. Financial support: none.}

Authors' contribution: N.M.F. vG. collected and analyzed the data and wrote the draft of the paper; A.A. vdE. and S.D.P. performed the blood analysis; H.L.Z. performed analysis of the control group; Y.S. dB.: contributed to the design of the study and critically reviewed the paper; B.I.W.: performed the statistical analysis; C.M.J. vN. and C.J.J.M. critically reviewed the paper; G.B. performed acquisition of data and critically reviewed the paper; R.A.dM. contributed to the design of the study and critically reviewed the paper. All authors have approved the final version of the manuscript.

Acknowledgements: Dutch Autoimmune Hepatitis Study Group: J Drenth (Radboud University, Nijmegen), J den Ouden (Haga hospital, Den Haag), R Verdonk (St Antonius hospital Nieuwegein), J Vrolijk (Rijnstate hospital), G Koek (Maastricht medical center), J Brouwer (Renier de graaf hospital Delft), L Baak (Onze Lieve Vrouwe Gasthuis, Amsterdam), B van Hoek (Leiden University Medical Center, Leiden), A Baven-Pronk (Leiden University Medical Center, Leiden), M Coenraad (Leiden University Medical Center, Leiden), K van Erpecum (University Medical center Utrecht, Utrecht), U Beuers (Academic Medical Center, Amsterdam), M Klempt-Kropp (Medisch Centrum Alkmaar, Alkmaar), J van Meyel (Sint Lucas Andreas Ziekenhuis, Amsterdam), R Linskens (St. Anna ziekenhuis, Geldrop), B Spanier (Rijnstate Ziekenhuis, Arnhem), J Kneppelhout (St. Anna ziekenhuis, Geldrop), J Kuyvenhoven (Kennemer Gasthuis, Haarlem), E van Geenen (Bronovo ziekenhuis, den Haag), M Wagtmans (Rode Kruis ziekenhuis, Beverwijk), D Cahen 
(Ziekenhuis Amstelland, Amstelveen), F Wolfhagen (Tweesteden ziekenhuis, Tilburg), P Kingma (Tergooiziekenhuizen, Hilversum), J de Vree (Medisch Centrum Leeuwarden, Leeuwarden), R Loffeld (Zaans Medisch Centrum, Zaandam), $\mathrm{H}$ van Buuren (Erasmus University Medical Center, Rotterdam), P Friederich (Meander Medisch Centrum, Amersfoort), T Schreuder (Slingeland ziekenhuis, Doetichem), A van Milligen de Wit (Amphia ziekenhuis, Breda), M Alleman (Isala, Zwolle), A Bhalla (Hagaziekenhuis, Den Haag), P Stadhouders (St. Antonius ziekenhuis, Nieuwegein), M Verhagen (Diakonessenhuis, Utrecht), C Copra (Erasmus University Medical Center, Rotterdam), M Pronk (Erasmus University Medical Center, Rotterdam).

\section{REFERENCES}

1. Manns MP, Czaja AJ, Gorham JD, et al. Diagnosis and management of autoimmune hepatitis. Hepatology 2010; 51: 2193-2213. doi: 10.1002/ hep. 23584

2. Alvarez F, Berg PA, Bianchi FB, et al. International Autoimmune Hepatitis Group Report: review of criteria for diagnosis of autoimmune hepatitis. J Hepatol 1999; 31: 929-938. doi: 10.1016/S0168-8278(99)80297-9

3. Hennes EM, Zeniya M, Czaja AJ, et al. Simplified criteria for the diagnosis of autoimmune hepatitis. Hepatology 2008; 48: 169-176. doi: 10.1002/hep.22322

4. Singh G, Palaniappan S, Rotimi O, Hamlin PJ. Autoimmune hepatitis triggered by hepatitis A. Gut 2007; 56: 304. doi: 10.1136/gut.2006.111864

5 Vento S, Cainelli F. Is there a role for viruses in triggering autoimmune hepatitis? Autoimmun Rev 2004; 3: 61-69. doi: 10.1016/S15689972(03)00053-3

6. Castellote J, Guell E, Porta F. Autoimmune hepatitis following cytomegalovirus infection. Med Clin (Barc) 2001; 117: 76.

7. Nobili V, Comparcola D, Sartorelli MR, Devito R, Marcellini M. Autoimmune hepatitis type 1 after Epstein-Barr virus infection. Pediatr Infect Dis J 2003; 22: 387.

8. Nagasaki F, Ueno Y, Mano Y, et al. A patient with clinical features of acute hepatitis E viral infection and autoimmune hepatitis. Tohoku J Exp Med 2005; 206: 173-179. doi: 10.1620/tjem.206.173

9. Vieira CL, Baldaia C, Fatela N, Ramalho F, Cardoso C. Case of acute hepatitis E with concomitant signs of autoimmunity. World J Hepatol 2013; 5: 152-155. doi: 10.4254/wjh.v5.i3.152

10. Iakimchuk KS, Malinnikova EI, Poleshchuk VF, Mikhailov MI. Role of hepatitis $\mathrm{A}$ and $\mathrm{E}$ viruses in the development of autoimmune diseases. Vopr Virusol 2011; 56: 27-29.

11. Dalton HR, Pas SD, Madden RG, van der Eijk AA. Hepatitis e virus: current concepts and future perspectives. Curr Infect Dis Rep 2014; 16: 399. doi: 10.1007/s11908-014-0399-8

12. Pischke S, Suneetha PV, Baechlein C, et al. Hepatitis E virus infection as a cause of graft hepatitis in liver transplant recipients. Liver Transpl 2010; 16: 74-82. doi: 10.1002/lt.21958

13. Pischke S, Gisa A, Suneetha PV, et al. Increased HEV seroprevalence in patients with autoimmune hepatitis. PLoS One 2014; 9: e85330. doi: 10.1371/journal.pone.0085330
14. van Gerven NM, Verwer BJ, Witte BI, et al. Epidemiology and clinical characteristics of autoimmune hepatitis in the Netherlands. Scand J Gastroenterol 2014; 49: 1245-1254. doi: 10.3109/00365521.2014.946083

15. Slot E, Hogema BM, Riezebos-Brilman A, Kok TM, Molier M, Zaaijer HL. Silent hepatitis E virus infection in Dutch blood donors, 2011 to 2012. Euro Surveill 2013; 18. doi: 10.2807/1560-7917. ES2013.18.31.20550

16. Pas SD, de Man RA, Mulders C, et al. Hepatitis E virus infection among solid organ transplant recipients, the Netherlands. Emerg Infect Dis 2012; 18: 869-872. doi: 10.3201/eid1805.111712

17. Baylis SA, Hanschmann KM, Blumel J, Nubling CM; HEV Collaborative Study Group. Standardization of hepatitis E virus (HEV) nucleic acid amplification technique-based assays: an initial study to evaluate a panel of HEV strains and investigate laboratory performance. J Clin Microbiol 2011; 49: 1234-1239. doi: 10.1128/JCM.02578-10

18. Alvarez F, Berg PA, Bianchi FB, et al. International Autoimmune Hepatitis Group Report: review of criteria for diagnosis of autoimmune hepatitis. J Hepatol 1999; 31: 929-938. doi: 10.1016/S0168-8278(99)80297-9

19. Le Cann P, Tong MJ, Werneke J, Coursaget P. Detection of antibodies to hepatitis $\mathrm{E}$ virus in patients with autoimmune chronic active hepatitis and primary biliary cirrhosis. Scand J Gastroenterol 1997; 32: 387-389. doi: 10.3109/00365529709007689

20. Verhoef L, Koopmans M, Duizer E, Bakker J, Reimerink J, Van Pelt W. Seroprevalence of hepatitis E antibodies and risk profile of HEV seropositivity in The Netherlands, 2006-2007. Epidemiol Infect 2012; 140: 1838-1847. doi: 10.1017/S0950268811002913

21. Mansuy JM, Bendall R, Legrand-Abravanel F, et al. Hepatitis E virus antibodies in blood donors, France. Emerg Infect Dis 2011; 17: 23092312. doi: 10.3201/eid1712.110371

22. Pas SD, Streefkerk RH, Pronk M, et al. Diagnostic performance of selected commercial HEV IgM and IgG ELISAs for immunocompromised and immunocompetent patients. J Clin Virol 2013; 58: 629-634. doi: 10.1016/j.jcv.2013.10.010

23. Eder M, Beinhardt S, Strassl R, et al. High seroprevalence of hepatitis E in patients with autoimmune hepatitis. J Hepatol 2015; 62: S790. doi: 10.1016/S0168-8278(15)31361-1

24. Dalton HR, Stableforth W, Thurairajah P, et al. Autochthonous hepatitis E in Southwest England: natural history, complications and seasonal variation, and hepatitis E virus IgG seroprevalence in blood donors, the elderly and patients with chronic liver disease. Eur J Gastroenterol Hepatol 2008; 20: 784-790. doi: 10.1097/ MEG.0b013e3282f5195a

25. Vogel A, Manns MP, Strassburg CP. Autoimmunity and viruses. Clin Liver Dis 2002; 6: 739-753.

26. Kamar N, Bendall RP, Peron JM, et al. Hepatitis E virus and neurologic disorders. Emerg Infect Dis 2011; 17: 173-179. doi: 10.3201/ eid1702.100856

27. van den Berg B, van der Eijk AA, Pas SD, et al. Guillain-Barre syndrome associated with preceding hepatitis E virus infection. Neurology 2014; 82: 491-497. doi: 10.1212/WNL.0000000000000111 